\title{
Primary endobronchial liposarcoma successfully resected via bronchoscopy: A rare case report with genetic analysis
}

\author{
YUANSHUN LIU ${ }^{1,2^{*}}$, HUA JIANG ${ }^{1,2^{*}}$, QIURAN XU ${ }^{3}$, HONGBIN ZHOU $^{1,3}$ and YAQING LI ${ }^{1,3}$ \\ ${ }^{1}$ Department of Respiratory Medicine, Zhejiang Provincial People's Hospital, People's Hospital of Hangzhou Medical College, \\ Hangzhou, Zhejiang 310014; ${ }^{2}$ The Second Clinical Medical College, Zhejiang Chinese Medical University, \\ Hangzhou, Zhejiang 310053; ${ }^{3}$ Key Laboratory of Tumor Molecular Diagnosis and Individualized Medicine of \\ Zhejiang Province, Zhejiang Provincial People's Hospital, People's Hospital of \\ Hangzhou Medical College, Hangzhou, Zhejiang 310014, P.R. China
}

Received October 18, 2017; Accepted May 3, 2018

DOI: $10.3892 /$ ol.2018.8737

\begin{abstract}
Liposarcoma is the most common type of soft-tissue sarcoma, and predominantly originates from the extremities and retroperitoneal cavity. However, primary endobronchial liposarcoma is extremely rare. The present study reports on the case of a 54-year-old man, a smoker, diagnosed with primary endobronchial atypical lipomatous tumor/well-differentiated liposarcoma (ALT-WDLS), which was successfully resected via bronchoscopy. Chest computed tomography (CT) revealed a neoplasm in the left main bronchus measuring $12.8 \times 7.8 \mathrm{~mm}$. Bronchoscopy demonstrated multiple roundish, pedunculated, polypoid masses in the left main bronchus. Bioptic specimens were obtained from the roundish masses, and pathological examination revealed an adipose tissue-derived tumor. The masses were completely resected during the second bronchoscopy under general anesthesia. Histopathological examination of the radical resection specimen revealed that it was an ALT-WDLS. The patient showed good quality of life at the 6-month postoperative follow-up without evidence of recurrence. Immunohistochemistry was completed with the human homologue of murine double-minute type 2 (MDM2) (+), cyclin-dependent kinase 4 (CDK4) (+), p16 (+), S-100 (+), Ki-67 (+), cluster of differentiation 34 (CD34) (+) and retinoblastoma protein $(+)$, confirming ALT-WDLS. However, the fluorescence in situ hybridization assay revealed no amplification of MDM2 and CDK4 in the ALT-WDLS. To
\end{abstract}

Correspondence to: Dr Yaqing Li, Department of Respiratory Medicine, Zhejiang Provincial People's Hospital, People's Hospital of Hangzhou Medical College, 158 Shangtang Road, Hangzhou, Zhejiang 310014, P.R. China

E-mail: lidoctor03@126.com

${ }^{*}$ Contributed equally

Key words: primary endobronchial liposarcoma, atypical lipomatous tumor/well-differentiated liposarcoma, bronchoscopic surgery, murine double-minute type 2, cyclin-dependent kinase 4 the best of our knowledge, the present case report is the first to describe the clinicopathologic features and genetic analysis of endobronchial liposarcoma. Although rare, this case is a reminder that clinicians should consider the possibility of this rare endobronchial tumor in patients with nonspecific symptoms including chronic cough, chest pain and recurrent pneumonia. As in this case, endoscopic treatment provided an excellent clinical outcome in patients with primary endobronchial ALT-WDLS.

\section{Introduction}

Liposarcoma is the most common type of soft-tissue sarcoma, accounting for $15 \%$ of all sarcomas, with an incidence of 0.9 cases/100,000 person-year in adults (1). According to the 2002 World Health Organization classification, liposarcomas are classified into 4 histological subtypes: atypical lipomatous tumor/well-differentiated liposarcoma (ALT-WDLS), dedifferentiated liposarcoma, myxoid round cell liposarcoma, and pleomorphic liposarcoma (2). Liposarcoma may occur anywhere in the body and usually arises from the extremities (75\%) and retroperitoneal cavity (20\%) (3). However, primary tracheobronchial tumors are rare, accounting for only $0.6 \%$ of all lung tumors (4). Most of them arise from the tracheobronchial surface epithelium, and squamous cell carcinomas are the most common histological types (5). Compared to tracheobronchial epithelial tumors, mesenchymal neoplasms are quite rare and mostly benign. Endobronchial liposarcoma is an extremely rare malignant tumor. Herein, we present a case of primary endobronchial liposarcoma in the left main bronchus causing cough, left-sided chest pain, and polypnea after physical activity. The tumor was completely and successfully removed using high-frequency electric snare and argon plasma coagulation via flexible bronchoscopy.

Human homologue of the murine double-minute type 2 (MDM2) and cyclin-dependent kinase 4 (CDK4), located on chromosome 12q13-15, are believed to be the proto-oncogenes of liposarcoma and are thus used for diagnostic and therapeutic considerations (6-9). Immunohistochemical (IHC) analysis was performed to detect the levels of MDM2 and CDK4. Fluorescence in situ hybridization (FISH) assay was also 
performed to detect MDM2 and CDK4 gene amplification in liposarcoma tissue samples to further confirm the diagnosis and for therapeutic considerations. To the best of our knowledge, this is the first case of endobronchial ALT-WDLS that was assessed and diagnosed using the protein and DNA levels of MDM2 and CDK4.

\section{Materials and methods}

Immunohistochemistry. Formalin-fixed, paraffin-embedded (FFPE) tissues were cut into $4-\mu \mathrm{m}$ sections and mounted on glass slides for IHC and FISH analysis. The sections were routinely de-waxed, rehydrated in graded ethanol, and subjected to high-pressure antigen retrieval in $10 \mathrm{mM}$ sodium citrate ( $\mathrm{pH}$ 6.0). The sections were then incubated with $3 \%$ hydrogen peroxide to block endogenous peroxidase and $10 \%$ normal goat serum to block nonspecific binding. Then, the slides were incubated at $4^{\circ} \mathrm{C}$ overnight with a primary antibody (Table I). Primary mouse or rabbit monoclonal antibodies were purchased from ZSGB-Bio (Beijing, China). Following incubation with a biotin-labeled secondary antibody, horseradish peroxidase-streptavidin was added. Finally, sections were treated with DAB reagent and then counterstained with hematoxylin. Negative control slides were incubated in phosphate buffer saline without primary antibodies for each staining.

The IHC evaluation of each protein was performed independently by two professional pathologists in a double-blinded manner. In case of ambiguity, a consensus score was obtained for further evaluation. Five different fields at x400 magnification were randomly selected per slide. Staining intensity was classified as: 0, no staining; 1, weak staining=light yellow; 2, moderate staining=yellow brown; and 3, strong staining=brown. Percentages of positively stained cells were categorized as follows: $0, \leq 5 \%$ positive cells; $1,6-25 \%$ positive cells; $2,26-50 \%$ positive cells; $3,51-75 \%$ positive cells; and $4,>76 \%$ positive cells. The overall score was calculated using the following formula: staining intensity score $\mathrm{x}$ positive percentage score, which ranged from 0 to 12 . The overall score was graded as follows: - , negative; +, 1-4; ++, 5-8; and +++, 9-12.

FISH assay. FISH was performed on 4- $\mu$ m-thick FFPE tissue sections with MDM2 (12q15)/chromosome 12 centromere (CEP12) or CDK4 (12q13-14)/CEP12 dual-color probes (ZytoVision, Bremerhaven, Germany) following the manufacturer's instructions. At least 200 non-overlapping nuclei selected randomly were evaluated. A MDM2/CEP12 or $\mathrm{CDK} 4 / \mathrm{CEP} 12$ ratio $\geq 2$ was defined as amplification of MDM2 or CDK4.

Ethical statement. Written informed consent was obtained from the patient for publication of this case report and associated images. The study was approved by the Ethics Committee of Zhejiang Provincial People's Hospital.

\section{Case report}

A 54-year-old man who smoked was referred to Zhejiang Provincial People's Hospital (Hangzhou, China) on February 3 , 2017 with a 1-year history of cough and polypnea after physical activity, which worsened 3 days prior to consultation. The
Table I. Antibodies used in immunohistochemical staining.

\begin{tabular}{llcc}
\hline Antibody & \multicolumn{1}{c}{ Clone } & Dilution & Expression \\
\hline MDM2 & SMP14 & $1: 100$ & +++ \\
CDK4 & EP180 & $1: 100$ & +++ \\
p16 & ABM51100-10 & $1: 100$ & +++ \\
S-100 & 15E2E2+4C4.9 & $1: 100$ & ++ \\
Ki-67 & EP5 & $1: 100$ & ++ \\
CD34 & QBEnd/10 & $1: 100$ & +++ \\
Rb & 13A10 & $1: 50$ & ++ \\
\hline
\end{tabular}

MDM2, human homologue of murine double-minute type 2; CDK4, cyclin-dependent kinase 4; CD34, cluster of differentiation 34; Rb, retinoblastoma protein.

patient developed left-sided chest pain and fever (maximum temperature in the 3 days before admission: $38.3^{\circ} \mathrm{C}$ ). $\mathrm{He}$ denied hypertension, diabetes, cardiomyopathy, hepatopathy, nephropathy, and a family history of malignancy. The electrocardiogram and serum tumor markers were normal. Blood examination showed elevated inflammatory markers (leukocyte count, 20.2×10\%/1; neutrophil percentage, $85 \%$; and C-reactive protein, $117 \mathrm{mg} / \mathrm{l})$.

A chest X-ray revealed a large shadow in the left middle and inferior lung and abnormal lesions along the left pleura. Chest computed tomography (CT) scan (Siemens Healthcare, Forchheim, Germany) revealed a neoplasm in the left main bronchus, measuring $12.8 \times 7.8 \mathrm{~mm}$ at its greatest dimension, obstructive pneumonia, atelectasis of left lung, and a small amount of pleural effusion in the left hemithorax (Fig. 1).

Flexible bronchoscopy under local anesthesia was performed and demonstrated multiple roundish, pedunculated, polypoid masses in the left main bronchus, which almost occluded the bronchial lumen (Fig. 2A). Bioptic specimens were obtained from the roundish masses and pathological examination revealed an adipose tissue-derived tumor (Fig. 2B). The masses were covered with monolayer bronchial mucosal epithelium that was in good shape. Submucosal fibrofatty tissue hyperplasia and myxoid degeneration were seen.

No evidence of tumor metastasis was noted. The masses were completely resected during the second bronchoscopy under general anesthesia. The bronchoscope was introduced via an oral trachea cannula to remove the large bulk of the biopsy specimens. The mass was snared and resected at its base using a high-frequency electric snare. Subsequently, the tip of the argon plasma probe was placed in contact with the mass that was then extracted simultaneously with the frozen specimens. Intriguingly, 3 tumors were observed and resected completely and successfully in 3 pieces (Fig. 2C). Gross examination showed that the surgical specimens consisted of 3 solid masses with moderate hardness and gray cut surfaces, measuring $2.8 \times 1.5 \times 1.5,1.7 \times 0.7 \times 1$, and $1.3 \times 0.8 \times 0.6 \mathrm{~cm}$. Histopathological examination of the radical resection specimen revealed that it was an ALT-WDLS. Microscopically, the tumor was composed of mature lipocytes with varying sizes and hyperplasia lipoblasts that had large, atypical, and hyperchromatic nuclei (Fig. 2D). 

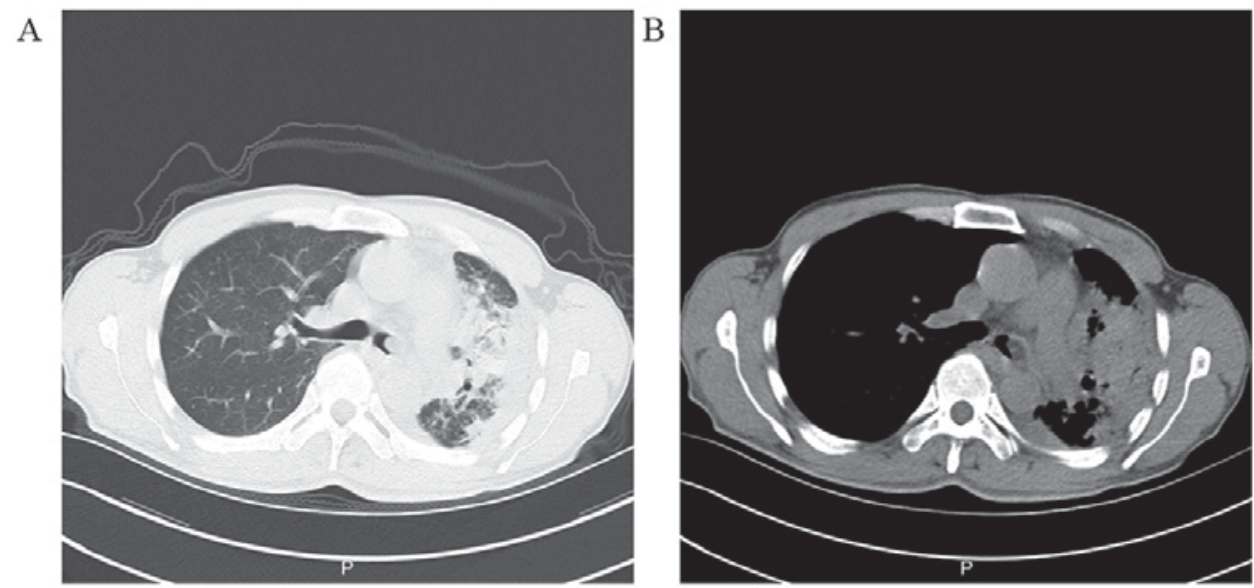

Figure 1. Chest $\mathrm{CT}$ scan demonstrating a roundish mass measuring $12.8 \times 7.8 \mathrm{~mm}$ in the left main bronchus and obstructing the bronchial lumen. (A) Lung window. (B) Mediastinal window. CT, computed tomography.
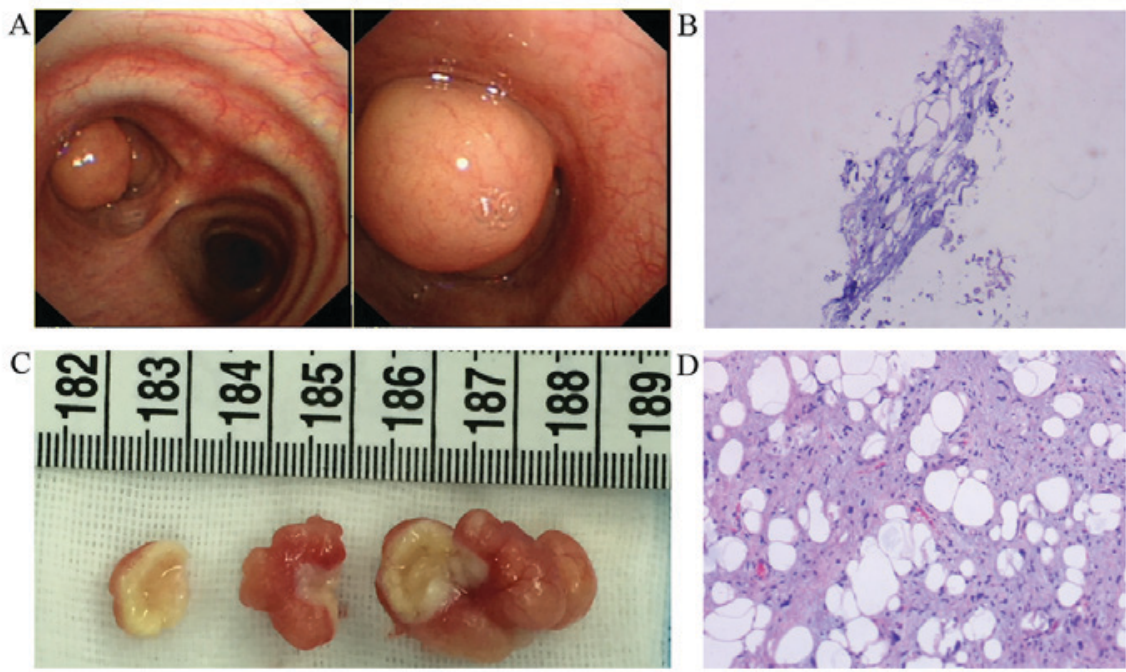

Figure 2. (A) The bronchoscopy exhibited multiple roundish, pedunculated, polypoid masses in the left main bronchus, which almost occluded the bronchial lumen. (B) Histopathological examination of the bioptic specimens revealed an adipose tissue-derived tumor (hematoxylin and eosin staining; original magnification, x 100). (C) Gross appearance of the 3 tumors following resection using high-frequency electric snare and argon plasma coagulation. (D) Histopathological images of radical resection specimens. The atypical lipomatous tumor/well-differentiated liposarcoma was composed of mature lipocytes with varying sizes and hyperplasia lipoblasts that had large, atypical and hyperchromatic nuclei (hematoxylin and eosin staining; original magnification, x100).

After appropriate antibiotic therapy and endoscopic surgery, the patient recovered uneventfully. Chest CT performed 1 month postoperative did not show any endobronchial lesion (Fig. 3A and B). In the 6-month follow-up, bronchoscopy revealed normal bronchial mucosa, and the patient has good quality of life without evidence of recurrence (Fig. 3C and D).

We analyzed FFPE tissues from surgically resected endobronchial liposarcoma specimens to determine the protein levels of MDM2 and CDK4 by IHC and DNA amplification of MDM2 and CDK4 via FISH. The details on the antibodies used and IHC results are presented in Table I. IHC was completed on all 3 resected specimens with the human homologue of MDM2 (+), CDK4 (+), p16 (+), S-100 (+), Ki-67 (+), CD34 (+), and $\mathrm{Rb}(+)$, confirming ALT-WDLS (Fig. 4A-G). FISH assay performed on all 3 resected specimens found no amplification of MDM2 and CDK4 in the ALT-WDLS (Fig. 4H and I).

\section{Discussion}

Liposarcoma is the most common soft tissue malignancy and represents approximately $20 \%$ of newly diagnosed cases (10). It may occur anywhere in the body and usually arises from the extremities (75\%) and retroperitoneal cavity (20\%) (3). We herein report a case of a man who presented with the disease at 54 years of age, which was consistent with the peak incidence of liposarcoma at 50-65 years of age (9). To the best of our knowledge, the present report is the first to describe the clinicopathologic features and perform genetic analysis of endobronchial liposarcoma. Although rare, this case is a reminder that clinicians should consider the possibility of this rare endobronchial tumor in patients with nonspecific symptoms such as chronic cough, chest pain, and recurrent pneumonia. Our case was initially misdiagnosed as chronic bronchitis in other hospitals. Conventional plain chest 

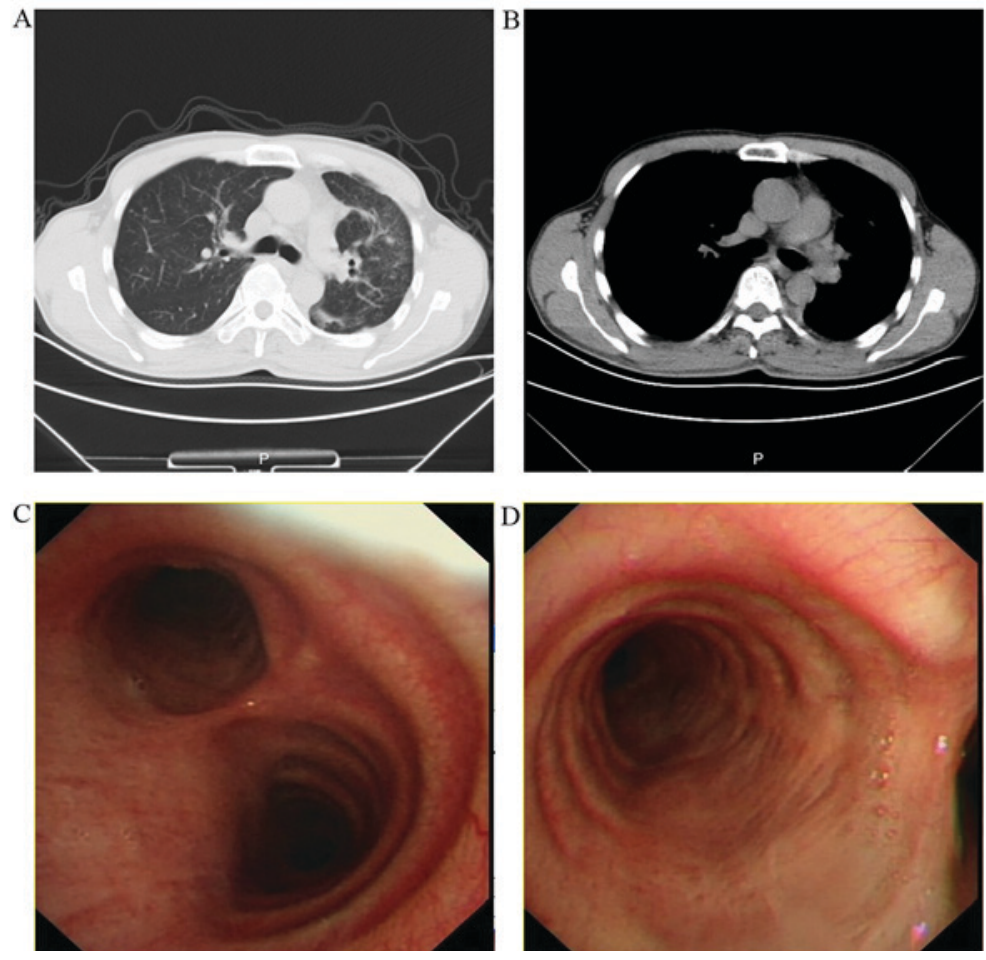

Figure 3. (A and B) Chest CT performed 1-month postoperatively did not show any endobronchial lesion. The left-sided pneumonia was improved, and pleural effusion was reduced when compared with the preoperative chest CT findings. (C and D) Bronchoscopy performed 6-month postoperatively revealed normal bronchial mucosa in the left main bronchus. CT, computed tomography.
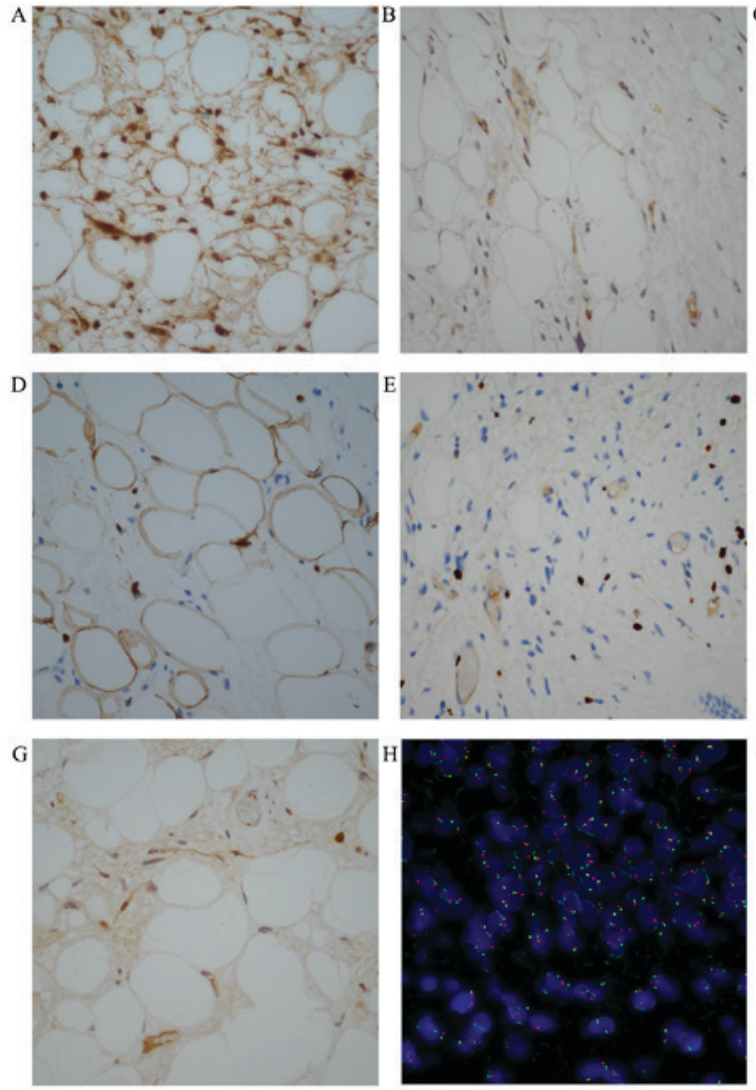
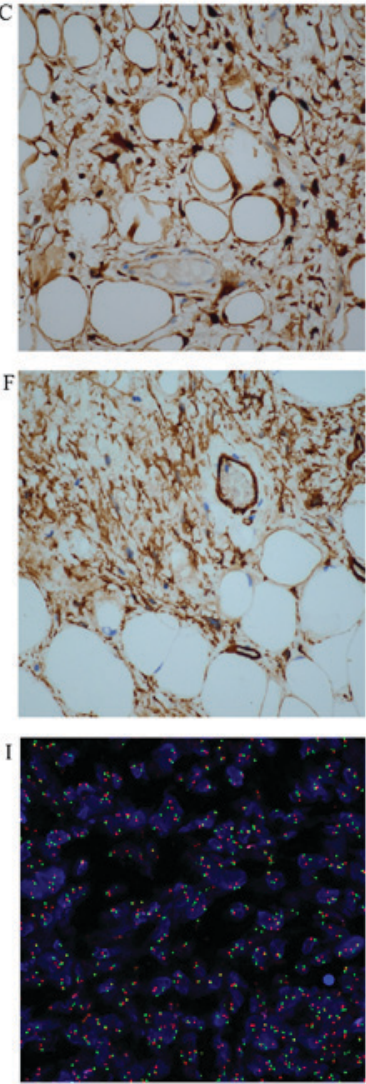

Figure 4. Representative immunohistochemistry images of the radical resection specimens (original magnification, x400). (A) MDM2, (B) CDK4, (C) p16, (D) S-100, (E) Ki-67, (F) CD34 and (G) Rb. The FISH assay revealed non-amplification of MDM2 and CDK4. (H) The ratio of MDM2 (red signal) to CEP12 (green signal) was 1.03 (original magnification, x1,000). (I) The ratio of CDK4 (red signal) to CEP12 (green signal) was 1.06 (original magnification, x1,000). MDM2, murine double-minute type 2; CDK4, cyclin-dependent kinase 4; FISH, fluorescence in situ hybridization; CD34, cluster of differentiation 34; Rb, retinoblastoma protein; CEP12, chromosome 12 centromere. 
radiographs have little diagnostic value for tracheobronchial tumors. Chest CT and bronchoscopy should be performed early to determine any tracheobronchial tumor. The tumor biopsy specimens obtained via bronchoscopy may be insufficient for histological examination, while endoscopic radical resection specimens can make a definitive diagnosis (11). In our present study, the pathologic result of bioptic specimens was adipose tissue-derived tumor, whereas that of endoscopic radical resection specimens was ALT-WDLS. The present case highlights the importance of diagnosis standards for the pathologic diagnosis of endobronchial liposarcoma. Liposarcoma is relatively resistant to chemotherapy and radiotherapy (12). The management of primary endobronchial ALT-WDLS, particularly the choice between endoscopic treatment and radical surgery, is controversial because of the disease rarity. As in this case, endoscopic treatment provides an excellent clinical outcome in patients with primary endobronchial ALT-WDLS. Our patient remains under careful long-term follow-up to preclude the possibility of local recurrence and distant metastasis.

ALT-WDLS is the predominant histopathologic subtype and represents $40-45 \%$ of all liposarcomas (13). A diagnosis of liposarcoma based solely on conventional histology may be difficult, and genetic and molecular analyses are helpful for diagnosis and targeted therapies $(9,10,14)$. A total of $90 \%$ of cases of ALT-WDLS show MDM2 and CDK4 amplification, which are located on chromosome 12q13-15 (9). MDM2 overexpression promotes cell cycle progression and tumor progression through ubiquitination and degradation of p53 (15-17). CDK4 has a fundamental role in oncogenesis by inactivating p53 and $\mathrm{Rb}$ phosphorylation $(9,13,18,19)$. CDK inhibitor $2 \mathrm{~A}(\mathrm{p} 16)$, an important tumor-suppressor gene, suppresses cell cycle progression through binding to CDK4/6 (20). He et al (21) previously suggested that p16 seemed to be a sensitive diagnostic biomarker for ALT-WDLS. It may be a valuable indication for distinguishing ALT-WDLS from deep-seated lipomas. Thway et al (22) stressed that p16 appeared to be useful in differentiating ALT-WDLS/dedifferentiated liposarcoma from other adipocytic neoplasms, which is consistent with the research of He et al (21). IHC for MDM2 and CDK4 can be used as a surrogate method in diagnosing WDLS and improving the diagnostic accuracy (7,23-25). Although cytogenetic analyses of MDM2 and CDK4 in liposarcomas of the extremities and retroperitoneal cavity have been performed, no research on MDM2 and CDK4 in endobronchial liposarcoma has been reported by far. Thway et al (22) indicated that the combinations of p16, MDM2, and CDK4 are useful tools for distinguishing ALT-WDLS/dedifferentiated liposarcoma from other adipocytic tumors. Kammerer-Jacquet et al (14) suggested that the combinations of p16, MDM2, and CDK4 might improve the diagnostic specificity of ALT-WDLS/dedifferentiated liposarcoma, which is agreement with the study of Thway et al (22). Immunostaining of p16, MDM2 and CDK4 proteins were intensively positive in the present case, confirming ALT-WDLS. However, FISH assay revealed non-amplification of MDM2 and CDK4 in the patient. Therefore, high levels of MDM2 and CDK4 proteins are not caused by gene amplification. This result is probably caused by other molecular regulation mechanisms such as transcriptional and post-transcriptional control of MDM2 and CDK4 gene expression. The array-based comparative genomic hybridization (array-CGH) technique has been successfully applied to detect chromosome aberrations and genomic imbalance in pleomorphic myxoid liposarcoma (26). Other assays such as array-CGH could be performed in this study which may provide more information about primary endobronchial liposarcoma. In addition, other genetic events such as gene mutations in CTNNB1, CDH1, FBXW7, EPHA1 and the fusion of FUS-DDIT3/EWSR1-DDIT3, known to be involved in liposarcomas could be performed. We acknowledge it as the limitation of our study. Therefore, reports of more such cases and further research are needed to elucidate the specific pathogenesis of primary endobronchial liposarcomas and whether it differs from liposarcomas in the extremity and retroperitoneum.

\section{Acknowledgements}

Not applicable.

\section{Funding}

The present study was supported by grants from the National Natural Science Foundation of China (grant nos. 81470241 and 81470109) and The Foundation of Science and Technology Department of Zhejiang Province (grant no. 2014C37022).

\section{Availability of data and materials}

All data generated or analyzed during this study are included in this published article.

\section{Authors' contribution}

YQL and YSL designed the study, and YSL and HJ collected the data and drafted the manuscript. QRX and HBZ performed the experiment and helped to revise the manuscript. All authors read and approved the final manuscript.

\section{Ethics approval and consent to participate}

The present study was approved by the Ethics Committee of Zhejiang Provincial People's Hospital. Written informed consent was obtained from the patient to participate in the study.

\section{Consent for publication}

Written informed consent was obtained from the patient for the publication of this case report and associated images.

\section{Competing interests}

The authors declare that they have no competing interests.

\section{References}

1. Ducimetière F, Lurkin A, Ranchère-Vince D, Decouvelaere AV Péoc'h M, Istier L, Chalabreysse P, Muller C, Alberti L, Bringuier PP, et al: Incidence of sarcoma histotypes and molecular subtypes in a prospective epidemiological study with central pathology review and molecular testing. PLoS One 6: e20294, 2011. 
2. Fletcher CDM, Bridge JA, Hogendoorn P and Mertens F (eds): WHO classification of tumours of soft tissue and bone. 4th edition. IARC Press, Lyon, 2013.

3. Kashu Y, Yukumi S, Tsunooka N, Tanigawa K, Arakane M, Nakagawa $\mathrm{H}$ and Kawachi K: Successful resection of a massive mediastinal liposarcoma that rapidly extended into the entire left thoracic cavity: Report of a case. Surg Today 42: 68-71, 2012.

4. Stevic R and Milenkovic B: Tracheobronchial tumors. J Thorac Dis 8: 3401-3413, 2016.

5. Macchiarini P: Primary tracheal tumours. Lancet Oncol 7: 83-91, 2006

6. Weaver J, Downs-Kelly E, Goldblum JR, Turner S, Kulkarni S, Tubbs RR, Rubin BP and Skacel M: Fluorescence in situ hybridization for MDM2 gene amplification as a diagnostic tool in lipomatous neoplasms. Mod Pathol 21: 943-949, 2008.

7. Dei Tos AP, Doglioni C, Piccinin S, Sciot R, Furlanetto A, Boiocchi M, Dal Cin P, Maestro R, Fletcher CD and Tallini G: Coordinated expression and amplification of the MDM2, CDK4, and HMGI-C genes in atypical lipomatous tumours. J Pathol 190: $531-536,2000$.

8. Singer S, Socci ND, Ambrosini G, Sambol E, Decarolis P, Wu Y, O'Connor R, Maki R, Viale A, Sander C, et al: Gene expression profiling of liposarcoma identifies distinct biological types/subtypes and potential therapeutic targets in well-differentiated and dedifferentiated liposarcoma. Cancer Res 67: 6626-6636, 2007.

9. Kollar A and Benson C: Current management options for liposarcoma and challenges for the future. Expert Rev Anticancer Ther 14: 297-306, 2014

10. Nassif NA, Tseng W, Borges C, Chen P and Eisenberg B: Recent advances in the management of liposarcoma. F1000Res 5: 2907, 2016.

11. Spinelli P, Pizzetti P, Lo Gullo C, Rocca F, Gobbi A and Ravasi G: Resection of obstructive bronchial fibrolipoma through the flexible fiberoptic bronchoscope. Endoscopy 14: 61-63, 1982.

12. Patel RB, Li T, Liao Z, Jaldeepbhai JA, Perera HAPNV, Muthukuda SK, Dhirubhai DH, Singh V, Du X and Yang J: Recent translational research into targeted therapy for liposarcoma. Stem Cell Investig 4: 21, 2017.

13. Laurino L, Furlanetto A, Orvieto E and Dei Tos AP. Well-differentiated liposarcoma (atypical lipomatous tumors). Semin Diagn Pathol 18: 258-262, 2001.

14. Kammerer-Jacquet SF, Thierry S, Cabillic F, Lannes M, Burtin F, Henno S, Dugay F, Bouzillé G, Rioux-Leclercq N, Belaud-Rotureau MA and Stock N: Differential diagnosis of atypical lipomatous tumor/well-differentiated liposarcoma and dedifferentiated liposarcoma: utility of p16 in combination with MDM2 and CDK4 immunohistochemistry. Hum Pathol 59: 34-40, 2017.

15. Vargas DA, Takahashi S and Ronai Z: Mdm2: A regulator of cell growth and death. Adv Cancer Res 89: 1-34, 2003.

16. Oliner JD, Kinzler KW, Meltzer PS, George DL and Vogelstein B: Amplification of a gene encoding a 553 -associated protein in human sarcomas. Nature 358: 80-83, 1992.
17. Oliner JD, Pietenpol JA, Thiagalingam S, Gyuris J, Kinzler KW and Vogelstein B: Oncoprotein MDM2 conceals the activation domain of tumour suppressor p53. Nature 362: 857-860, 1993

18. Kato J, Matsushime H, Hiebert SW, Ewen ME and Sherr CJ: Direct binding of cyclin D to the retinoblastoma gene product $(\mathrm{pRb})$ and $\mathrm{pRb}$ phosphorylation by the cyclin $\mathrm{D}$-dependent kinase CDK4. Genes Dev 7: 331-342, 1993.

19. Weinberg RA: The retinoblastoma protein and cell cycle control. Cell 81: 323-330, 1995.

20. Fåhraeus R, Paramio JM, Ball KL, Lain S and Lane DP. Inhibition of $\mathrm{pRb}$ phosphorylation and cell-cycle progression by a 20 -residue peptide derived from p16CDKN2/INK4A. Curr Biol 6: 84-91, 1996.

21. He M, Aisner S, Benevenia J, Patterson F, Aviv H and Hameed M p16 immunohistochemistry as an alternative marker to distinguish atypical lipomatous tumor from deep-seated lipoma. Appl Immunohistochem Mol Morphol 17: 51-56, 2009.

22. Thway K, Flora R, Shah C, Olmos D and Fisher C: Diagnostic utility of p16, CDK4, and MDM2 as an immunohistochemical panel in distinguishing well-differentiated and dedifferentiated liposarcomas from other adipocytic tumors. Am J Surg Pathol 36: 462-469, 2012.

23. Pilotti S, Della Torre G, Mezzelani A, Tamborini E, Azzarelli A, Sozzi G and Pierotti MA: The expression of MDM2/CDK4 gene product in the differential diagnosis of well differentiated liposarcoma and large deep-seated lipoma. Br J Cancer 82: $1271-1275,2000$

24. Binh MB, Sastre-Garau X, Guillou L, de Pinieux G, Terrier P, Lagacé R, Aurias A, Hostein I and Coindre JM: MDM2 and CDK4 immunostainings are useful adjuncts in diagnosing well-differentiated and dedifferentiated liposarcoma subtypes: A comparative analysis of 559 soft tissue neoplasms with genetic data. Am J Surg Pathol 29: 1340-1347, 2005.

25. Coindre JM, Mariani O, Chibon F, Mairal A, De Saint Aubain Somerhausen N, Favre-Guillevin E, Bui NB, Stoeckle E, Hostein I and Aurias A: Most malignant fibrous histiocytomas developed in the retroperitoneum are dedifferentiated liposarcomas: A review of 25 cases initially diagnosed as malignant fibrous histiocytoma. Mod Pathol 16: 256-262, 2003.

26. Creytens D, van Gorp J, Ferdinande L, Van Roy N and Libbrecht L: Array-based comparative genomic hybridization analysis of a pleomorphic myxoid liposarcoma. J Clin Pathol 67: 834-835, 2014

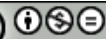

This work is licensed under a Creative Commons Attribution-NonCommercial-NoDerivatives 4.0 International (CC BY-NC-ND 4.0) License. 